\title{
A FUZZY MODEL UPDATING TECHNIQUE MOTIVATED BY BAYESIAN INFERENCE
}

\author{
A. Hanselowski, S. Ihrle, and M. Hanss \\ Institute of Engineering and Computational Mechanics \\ University of Stuttgart \\ Pfaffenwaldring 9, 70569 Stuttgart, Germany \\ e-mail: $\{$ andreas.hanselowski, sebastian.ihrle, michael.hanss\}@itm.uni-stuttgart.de
}

Keywords: Model updating, fuzzy arithmetic, Bayesian estimation, fuzzy Bayesian estimation, fuzzy prior

\begin{abstract}
In this paper, the problem of structural uncertainty of models in the field of model updating is discussed. It is shown that stochastic methods are not appropriate to quantify this type of uncertainty, because of its epistemic nature and different properties. In this context, the fuzzy Bayesian estimation is introduced, which enables the analysis of structural uncertainty by means of fuzzy arithmetic. In this framework, uncertain parameters of the model are estimated and represented by fuzzy numbers. The estimates are based on measured reference outputs and prior information about the parameters in form of a sparse sample of observations or expert knowledge. For this purpose, a methodology for the combination of the information of the reference data and the prior information is proposed. The additional information of the prior is beneficial, as the accuracy of the estimated parameters is increased.
\end{abstract}




\section{INTRODUCTION}

Traditional model updating techniques, as introduced by Friswell and Mottershead [7], are well established and widely used for the point estimation of parameters. However, the reference output for a complex system can rarely be matched precisely due to the underlying uncertainties in the problem. Thus, in addition to the point estimation, comprehensive solutions have been formulated which include the estimation of interval bounds of the parameters.

In this context, stochastic methods have been introduced for model updating, since here the point estimation as well as the intervals can be derived from the computed probability density function (PDF). The methods are well defined in the presence of stochastic uncertainties, where standard methods, such as maximum likelihood [6] and Bayesian estimation [23], are available. Whereas the maximum likelihood method enables an estimation of the parameters solely based on the reference output data, the Bayesian estimation additionally includes the prior information about the parameters. In fact, for model updating problems, prior information of the parameters is often available, in form of expert knowledge or the information from previous studies. The additional information is beneficial, as it improves the estimates and helps to reduce overfitting of the reference data [26]. Thus, Bayesian estimation is a very popular technique for model updating and parameter estimation in general.

Nevertheless, even though the method is suitable for problems with aleatory uncertainties, describing stochastic variations, it is not appropriate for epistemic uncertainties, resulting from a lack of information [24]. In particular, structural uncertainties, which result from simplification and idealization in modeling, are often immense for complex systems, as no sufficient knowledge of the underlying physical phenomena is available [16]. Furthermore, the available information is often present in form of a sparse sample of observations which is not sufficient to derive the underlying distribution. For this reason, the representation of this type of uncertainty by a PDF is not appropriate for the back-propagated reference outputs or the prior information about the parameters. Instead, those uncertainties are typically modeled by fuzzy sets or fuzzy numbers [12], which are well suited for modeling uncertainties originating from sparse data, deficient information, user intuition or expert knowledge. Moreover, those epistemic uncertainties must be computed differently, and here, in contrast to sampling techniques, like Markov chain Monte Carlo algorithm [8], interval or fuzzy methods [12] have proved to be appropriate.

In this context, the fuzzy Bayesian estimation is proposed in this paper, which is a method based on fuzzy arithmetic. The method combines the information in form of the back-propagated output uncertainty with the prior information in a Bayesian manner, but here the uncertainty is of epistemic type. The point estimation is based on least-squares estimation, as it is less restrictive on sparse data and is more appropriate for non-stochastic distributions. Uncertainty intervals resulting from model deficiencies are computed by the inverse fuzzy arithmetic [10]. Since the representation of the prior information by a PDF is controversial, for the fuzzy Bayesian estimation this information is encoded in a fuzzy number. In order to be able to merge prior information and the back-propagated information of the measured reference outputs, a framework is introduced which enables the representation of the combined information by a fuzzy number. Hereby, prior information about the parameters can be considered either as a sample of direct observations or as expert knowledge. The included prior is capable of improving the results, as the estimates are based on more information.

Nevertheless, it must be noted that a number of other methods have been proposed for the analysis of epistemic uncertainties. Interval-based methods have been introduced by Jaulin et al. [15] and Khodaparast et al. [18]. Fuzzy-arithmetic-based methods for model updating have 
been proposed by Liu et al. [22], Khodaparast et al. [17], and Erdogan et al. [5]. However, in these studies only the epistemic uncertainty of the reference outputs is propagated back to the parameters, essentially assuming no shortcomings of the model. Furthermore, for the estimation of the parameters, no prior information is considered.

As the methodology of uncertainty quantification is well established in the framework of statistics, the presented fuzzy Bayesian estimation will be compared to the classical Bayesian estimation. In this context, the terminology typically used in statistics is adopted, since it is very specific and allows for a convenient comparison of the stochastic methods and fuzzy methods.

The paper is structured as follows: In Section 2, the traditional inverse fuzzy arithmetic is outlined. Section 3 gives an overview over the Bayesian estimation in the stochastic framework. Section 4 introduces the fuzzy Bayesian estimation. In Section 5, the fuzzy Bayesian estimation is applied to a simple, yet exemplary overdetermined system. In Section 6, some final conclusions are drawn and open issues are discussed.

\section{Inverse fuzzy arithmetic}

The inverse fuzzy arithmetic (IFA) enables the computation of the uncertainty of parameters based on uncertainties of the model or the uncertainty originating from the variation of reference outputs. Thereby, the parameters are represented by fuzzy numbers, where $\alpha$-cuts describe the uncertainty intervals at different levels of membership $\mu=\alpha$. The method has been applied to a variety of inverse problems, which are subject to epistemic uncertainty, as presented in $[10,9]$. The steps that are necessary to compute the uncertain parameters are presented in the following.

1. A point estimation is performed, whereby a set of crisp parameters which fits best the reference data is found by an optimization technique.

2. Based on the deviation of the computed outputs from the first step and the measured reference data, the fuzzy output numbers are constructed.

3. An initial set of fuzzy numbers for the parameters is estimated and a forward analysis using the transformation method is performed. Hereby, for each $\alpha$-cut, gradients are computed, which enables a kind of system representation.

4. Based on the constructed system representation, the fuzzy output intervals from the second step are propagated back to the parameters.

\section{Bayesian estimation}

In Bayesian estimation, the stochastic distribution of the reference data and the distribution representing prior information of the parameters are combined into a single PDF. Thereby, the prior information about the parameters is represented by the prior $\operatorname{PDF} P(\boldsymbol{\theta})$. The distribution of the reference data is described by the likelihood function $\mathcal{L}(\boldsymbol{\theta} \mid \boldsymbol{y})=P(\boldsymbol{y} \mid \boldsymbol{\theta})$, which represents the likelihood of the parameters for a model with respect to the given reference information. For this purpose, the inverse problem must be solved, since the reference output distribution is given and one needs to find the distribution of the parameters in agreement with the output distribution. For this computation, often the Markov chain Monte Carlo algorithm [8] is applied. Both distributions are multiplied and normalized by the PDF $P(\boldsymbol{y})$ of the outputs, giving rise to the well-known Bayes equation 


$$
P(\boldsymbol{y} \mid \boldsymbol{\theta})=\frac{P(\boldsymbol{y} \mid \boldsymbol{\theta}) P(\boldsymbol{\theta})}{P(\boldsymbol{y})} .
$$

The resulting distribution $P(\boldsymbol{y} \mid \boldsymbol{\theta})$ is called posterior.

As mentioned above, the prior is often based on subjective belief, which represents information in form of expert knowledge, information from literature, previous studies, published work, researcher experience or intuition. For the prior PDF, often the normal distribution is assumed, which is justified by the maximum entropy principle [25]. It must be noted that only a proper definition of the prior leads to the superior result of the Bayesian framework. If the prior is estimated wrongly, its contribution will either be too small, having just little influence on the results, or in the other extreme, it will completely dominate the solution.

For the point estimation, in Bayesian statistics usually the minimum mean squared error estimator is used [20]. The estimator is the conditional expectation $\mathrm{E}\{\boldsymbol{y} \mid \boldsymbol{\theta}\}$ of the parameters, given the reference output. This corresponds to the mean of the posterior distribution.

In the context of Bayesian estimation, the bounds are defined in terms of credible intervals [2], which are taken from the posterior distribution. Thereby, the parameters are assumed to have crisp but unknown value, and the posterior describes the probability of the parameters taking a particular value.

However, from the perspective of classical statistical inference, the Bayesian prior is controversial. Prior information is typically available in form of epistemic belief about the parameters, which needs to be expressed by a PDF. In this context, it seems more appropriate to define the prior as a fuzzy quantity, as fuzzy representations are traditionally defined to model epistemic belief. In fact, several methods have been proposed, where the prior knowledge is defined in form of a fuzzy prior $[21,1]$. This has also motivated the fuzzy Bayesian estimation which will be introduced in the next section.

Another drawback of the Bayesian estimation is that it is not appropriate to quantify structural uncertainty. In this case, the reference outputs are crisp and the uncertainty emerges after the parameter update has been performed. The model is simply not capable of representing the reference outputs exactly, which gives rise to the uncertainty. In Bayesian estimation, however, only the uncertainty of the reference outputs can be propagated back to the parameters.

\section{Fuzzy Bayesian estimation}

In order to address the problems of the Bayesian estimation in the case of epistemic uncertainty, the fuzzy Bayesian estimation (FBE) is proposed. The method is motivated by Bayesian inference and is an extension of the already presented IFA. In the following, the method is discussed for its application to overdetermined systems where the reference output information as well as the prior information is present in form of a number of observations.

\subsection{Fuzzy prior}

Since the prior is often based on epistemic belief, a fuzzy prior is proposed which is in accordance with the definition of the fuzzy number. This enables the modeling of information available as epistemic belief in form of a discrete or continuous distribution. For this purpose, a flexible and consistent interface between the available epistemic information and its fuzzy representation is defined in form of percentage intervals. In the following, the framework is described for prior information in form of discrete samples. For this purpose, an example with 10 
direct observations of a parameter is considered. Obviously, the given information is deficient to some respect, as otherwise, one would not have to infer the parameters from the posterior, but solely rely on the prior information. The given sample does not satisfy the constraint on stochastic data, as it is neither generated randomly nor independent identically distributed [4]. Since the sample is also rather sparse and cannot be represented by a reasonable PDF, the information will be encoded in a fuzzy number based on percentage intervals, which describe the amount of the observations included in the interval. For example, for the membership value $\mu=0.0$ all 10 values are included, which gives rise to a $100 \%$-interval. As the membership values $\mu$ increase, less observations are incorporated. Thus, $\mu=0.2$ corresponds to an $80 \%$-interval, which includes 8 values, $\mu=0.4$ to an $60 \%$-interval including 6 values, and so on. For the membership level $\mu=1.0$, there are either no values included in case of an even number of observations, or one value in case of an odd number. For the odd number, the intervals slightly deviate from the real percentage of samples being included, but the representation asymptotically converges with increasing sample size.

In case of an even number of observations, there is one additional value which needs to be defined to represent the maximum value of the fuzzy number. For this purpose, the mean of the two values in the middle of the sample is taken as suggested in [27]. Note that the maximum of the resulting fuzzy number is the median of the sample and not the mean, since it is consistent with the idea of the percentage intervals. The discussed distribution and the corresponding fuzzy number is illustrated in Fig. 1. In case the prior information is present as epistemic belief, the representation needs to be reduced to a discrete sample in order to be able to express it in a fuzzy number.

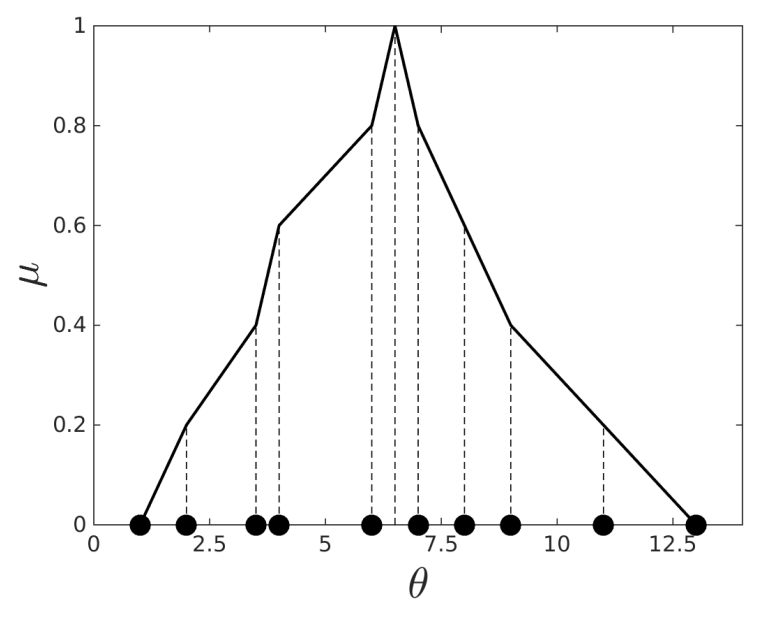

Figure 1: Definition of the fuzzy prior based on a discrete sample.

\subsection{Point estimation}

For the FBE, the additional information of the prior must be reflected in the point estimation. Here again, the discrete case is discussed, where a number of direct parameter observations and a number of output observations is available.

For the point estimation solely based on the reference outputs, the weighted least-squares estimator is chosen. The weights make sure that large output values do not dominate the solution by large absolute errors. According to the Markov-Gauss theorem [28], the least squares 
estimator has a number of beneficial properties, as it is less restrictive on sparse data and is better defined for distributions which are not independent identically distributed. It is more appropriate in the presence of epistemic uncertainties than other estimators, like for example the maximum likelihood estimator $[14,3]$.

For the point estimation of the fuzzy prior, simply the mean of the sample of observations is chosen. This is done for each parameter independently of each other. The estimate based on the reference data $\hat{\boldsymbol{\theta}}_{\mathbf{y}}$ is combined with the prior estimate $\hat{\boldsymbol{\theta}}_{\text {prior }}$ in a post-processing step

$$
\hat{\boldsymbol{\theta}}=n_{\mathrm{y}} \cdot \hat{\boldsymbol{\theta}}_{\mathbf{y}}+\lambda \cdot n_{\text {prior }} \cdot \hat{\boldsymbol{\theta}}_{\text {prior }} \cdot
$$

The two point estimates are multiplied by the sum $n$ of the observations available for each estimate, as each observation represents the evidence which supports it. Furthermore, if the contributions of the two estimates are different, that is if one observation of the output is less informative than one from the parameter or vice versa, then the prior can additionally be weighted with the factor $\lambda$. This representation is similar to the definition of the target function, which is often used for the point estimation for inverse problems. Here, $\lambda$ is the regularization parameter, and the prior is the regularization term.

As for the Bayesian estimation, the contribution of the two terms must be weighted appropriately, since otherwise, the results are not superior or even worse than solely based on the reference outputs. If the contributions cannot be judged, techniques such as L-Curve [11] and cross-validation [19] have to be utilized for the estimation of the regularization parameter $\lambda$.

In general, overdetermined systems are subject to bias error, since these models are not flexible enough to represent the data exactly. Therefore, the additional information of the prior is beneficial as the bias error is reduced. However, an overdetermined system might be illconditioned and thus become undetermined, as some of the reference outputs are uninformative. These systems can often represent the reference data accurately, but cannot generalize towards new system configurations, i.e. if the system setting is changed, they fail to predict the new outputs accurately. This phenomenon is known as overfitting, and in this case, the additional information provided by the prior helps to improve the condition of the problem. This is generally referred to as regularization and leads to better estimates of the parameters.

\subsection{Interval estimation}

The estimated intervals must also appropriately reflect the back-propagated reference information and the prior information according to their contribution. For this purpose, a procedure is proposed where the two fuzzy numbers are first reduced to two samples of discrete values before they are composed into the fuzzy posterior. Since the prior is constructed from discrete values, it can simply be decomposed again. For the back-propagated fuzzy number, however, a number of $\alpha$-cuts needs to be defined in order to reduce it to a sample. In fact, the back-propagated fuzzy number represents the discrete sample of reference outputs, since the maximum value reflects the mean of the sample and the worst-case intervals encompass all the reference values. Therefore, the fuzzy number can be reduced to a number of discrete values, according to the number of discrete observations of the reference outputs. After that, the discrete values of the back-propagated fuzzy number and the fuzzy prior can be combined into a single fuzzy number. This is done in the same manner as was previously presented for the prior fuzzy number in Fig. 1. The resulting $\alpha$-cuts of the fuzzy number can again be interpreted in terms of the information they include, based on percentage intervals. An illustrative example of this combination 
is given in Figs. 2 and 3. In Fig. 2, the two separate fuzzy numbers are reduced to the discrete samples and are then combined into one number in Fig. 3.

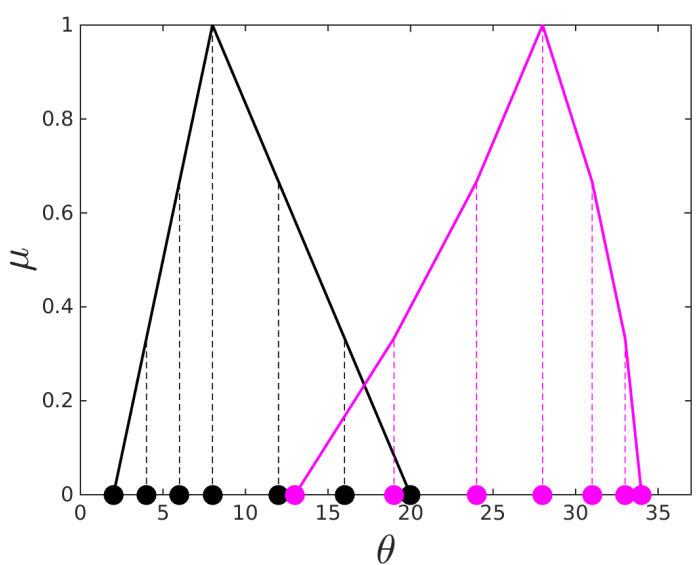

Figure 2: Back-propagated fuzzy number and the fuzzy prior number

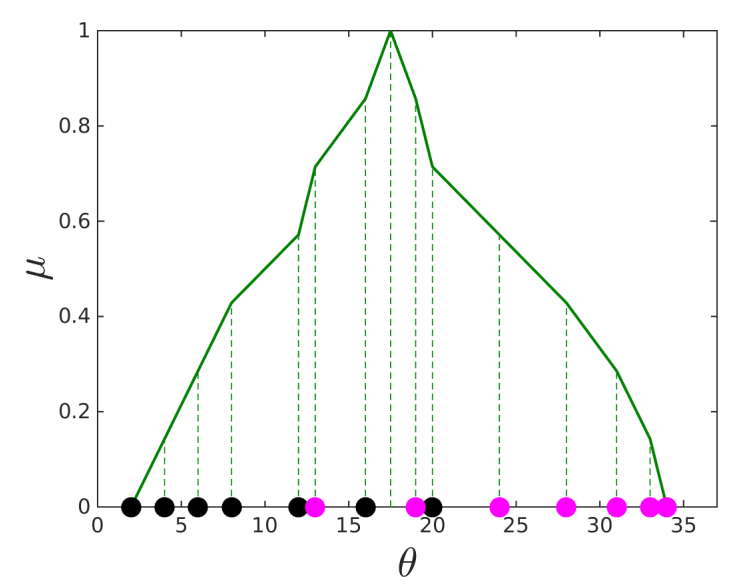

Figure 3: Fuzzy posterior combining the backpropagated fuzzy number and the fuzzy prior

In this representation, each observation is weighted equally. However, in the case of $\lambda \neq 1$, the contribution of the values of the inputs and the outputs needs to be altered in order to reflect the relative contribution. This can be done by changing the number of discrete values for the back-propagated fuzzy number and the fuzzy prior accordingly. For example, for $\lambda=0.6$, one could choose $3 \alpha$-cuts for the prior, which would give rise to 6 discrete values, and for the back-propagated fuzzy number one could choose $5 \alpha$-cuts resulting in 10 values. In both cases, the central value is neglected giving rise to an even number of samples.

It must be noted that the maximum of the fuzzy number corresponds to the median, and therefore, in general deviates from the point estimation. This is in analogy to statistics, where usually the mean value of a PDF is of interest for the point estimation, and not the maximum. Thus, in the representation chosen here, the intervals describe the percentage of information they include, and the point estimates reflect the most plausible value in accordance with the given information.

\section{Example}

As an example for the presented approach, the solution of overdetermined systems is considered, using an overdetermined linear system $\boldsymbol{A} \boldsymbol{\theta}=\boldsymbol{y}$ in particular [13]. Even though the system is trivial, it represents important aspects of the problem of structural uncertainty, which also holds for complex systems. Here, $\boldsymbol{\theta}$ represents the parameters to be updated, $\boldsymbol{A}$ the model, and $\boldsymbol{y}$ the reference outputs. In this case, the outputs $\boldsymbol{y}$ are considered to be crisp and the uncertainty results only from the deficient model.

In the following, an exemplary linear system is given and the problem is addressed in two settings. First, the problem is solved only by relying on the reference data, using IFA. Next, additional information in form of a prior $\boldsymbol{\theta}^{*}$ is provided, and the problem is solved by the FBE. The particular linear system considered is

$$
\left[\begin{array}{ll}
3.5 & 2.5 \\
1.4 & 2.0 \\
2.3 & 1.5
\end{array}\right] \cdot\left[\begin{array}{l}
\theta_{1} \\
\theta_{2}
\end{array}\right]=\left[\begin{array}{l}
2.6 \\
1.5 \\
2.6
\end{array}\right]
$$


The solution of the problem by the use of inverse fuzzy arithmetic is illustrated in the parameter space in Fig. 4. The gray lines indicate the solution for the three outputs independent of each other. Since there is no intersection of the three lines, the problem cannot be solved exactly and only an approximate solution can be found.

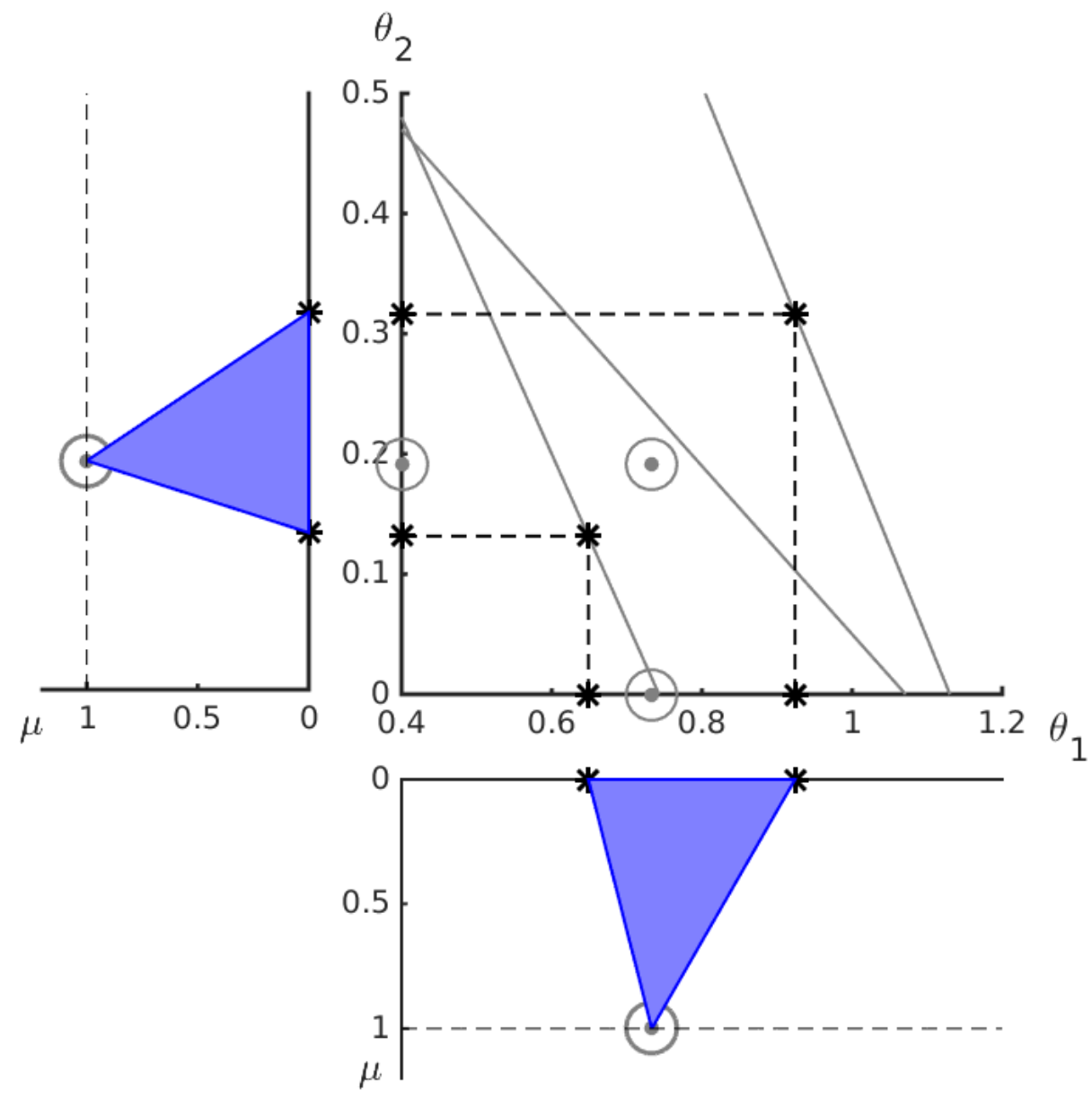

Figure 4: Worst-case interval estimation using inverse fuzzy arithmetic.

The point estimation, which is found by the suggested LSE, is indicated by the dot with the circle around it. This solution is also projected onto the two axes of $\boldsymbol{\theta}_{1}$ and $\boldsymbol{\theta}_{2}$. The worstcase bounds determined by the IFA are marked by the asterisks and are projected onto the two parameter axes via the dashed lines. Note that the asterisks lie on the two solution lines which are furthest away from the point estimation, as in the IFA just the conservative solution is of interest. The constructed fuzzy numbers for the two parameters are illustrated to the left and underneath the parameter space plot. As can be observed, the classical point estimation is taken for the maximum of the number, and the conservative bounds are represented by the worst-case intervals with the membership value zero.

In the following, prior information about the parameters $\theta^{*}$ is additionally included, where for each parameter three direct observations are given. The solution of the problem by the use of 
the FBE is illustrated in parameter space in Fig. 5. As the information about the prior parameters is assumed to be independent, it is represented by a square-shaped region in the parameter space which is confined by worst-case bounds. On the two axes, the worst-case bounds are denoted by black dots. The median, corresponding to the maximum of the prior fuzzy number, is indicated by the larger black dot. The mean value which represents the point estimation for the prior is depicted by a circle, and as can be observed, it lies in the direct vicinity of the median.

The combined point estimate, which incorporates the solution of the prior and the backpropagated information, is denoted by a square and is located on a line in between the two estimates. By changing the weighting though the $\lambda$-factor, the estimate would move along on this line. Since here an equal weighting of each observation is assumed, the combined estimate is half way in between.

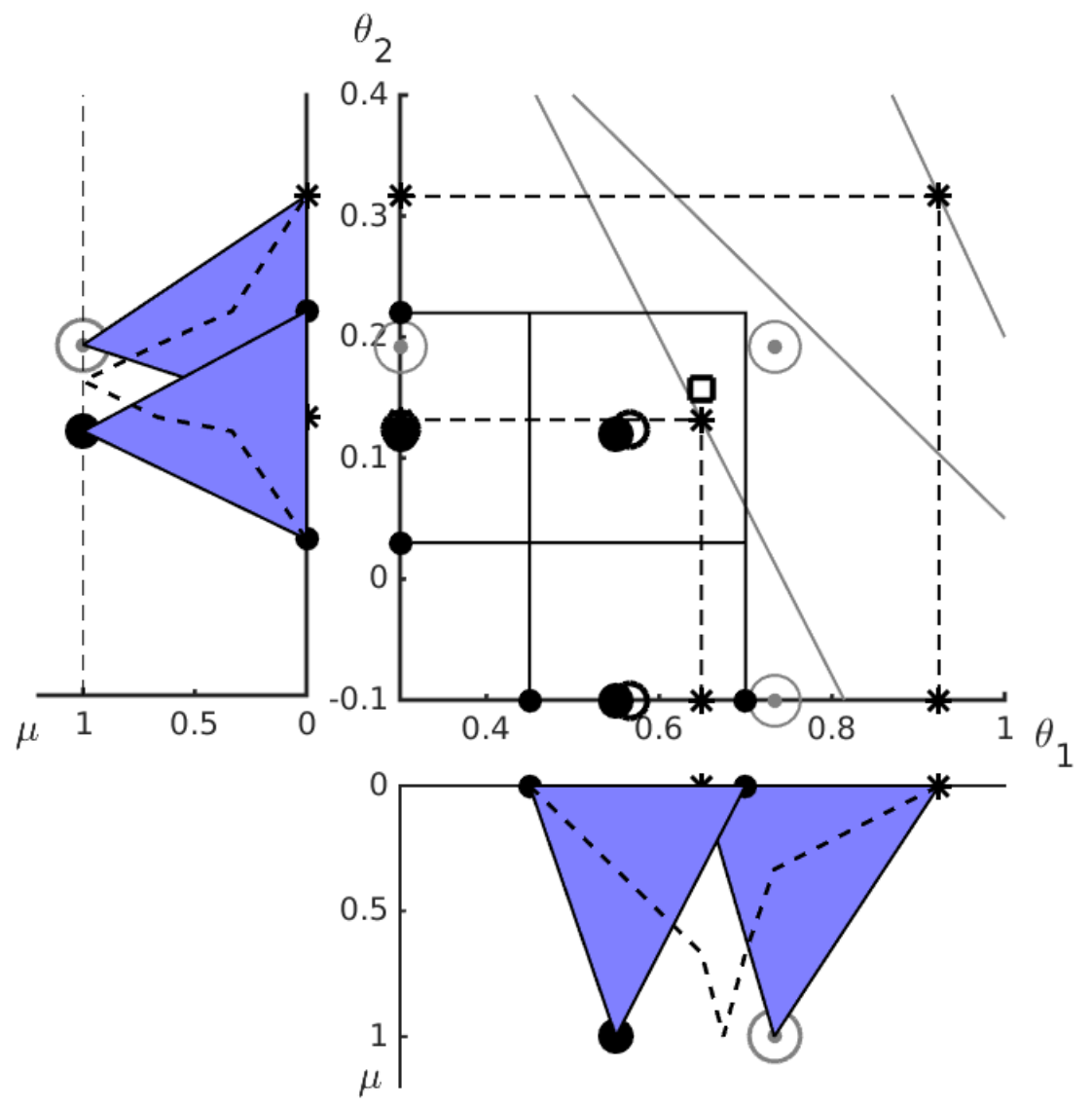

Figure 5: Problem illustration in parameter space for the fuzzy Bayesian estimation

The fuzzy priors and the back-propagated fuzzy numbers are combined into the fuzzy posterior for the estimation of the combined intervals, according to the definition above. For this purpose, the two fuzzy numbers are reduced to three discrete values, since there are three output observations and three parameter observations. As there is no relative weighting of the observations, that is $\lambda=1$, the 6 values are simply combined into one fuzzy number. In Fig. 5 the fuzzy posteriors are indicated by the dashed lines. 


\section{Conclusion and open issues}

In this paper, the problem of structural uncertainty in model updating is discussed, where the uncertainty results from the deficiency of the model in describing the investigated phenomenon in full detail. The underlying uncertainty is epistemic and must be handled differently as stochastic variations, since it exhibits other properties. Epistemic uncertainty is traditionally analyzed by fuzzy and interval arithmetic, and thus, the fuzzy Bayesian estimation is introduced in this paper. In this framework, the least-square estimator is used for the point estimation, since it is less restrictive on sparse data or not random distributions. For the interval estimation, inverse fuzzy arithmetic is used, which is a well established technique for inverse uncertainty quantification. Additionally, prior information about the parameters is included in the procedure in form of a fuzzy prior. The incorporation of the prior improves the results, as the estimates are based on more information. Moreover, the prior can be seen as a kind of regularization, which helps to deal with overfitting of reference data.

In order to be able to compute epistemic information by means of fuzzy arithmetic, a methodology is proposed which enables the transformation of the available information into a fuzzy number. The method allows the combination of information of the reference outputs and the prior information, available as a set of direct observations or as expert knowledge. This gives rise to the fuzzy posterior, which represents plausibility intervals of the estimated parameters.

The presented method excels Bayesian estimation in the presence of epistemic uncertainty, since it is less restrictive on sparse data and does not require the distribution to be random. Furthermore, fuzzy Bayesian estimation can applied to problems where the uncertainty is inherent in the model. The classical Bayesian estimation, on the other hand, is only appropriate for problems where the uncertainty is inherent to the reference outputs.

Nevertheless, this paper must be considered as an preliminary analysis, since many topics could not be described to full extend or have entirely been omitted. Instead, emphasis was placed on the comparison of the Bayesian estimation and the fuzzy Bayesian estimation and on the illustration of the drawbacks of the former approach in case of epistemic uncertainty. The fuzzy Bayesian estimation is worth being presented in more detail and being extended to different problems settings. Since here only discrete distributions have been considered, a proper analysis of uncertainties with continuous distributions needs to be performed additionally. Furthermore, in order to illustrate the full capability of the approach, the fuzzy Bayesian estimation will be applied to an elaborated model-updating problem in a future study.

\section{REFERENCES}

[1] B. Arnold and P. Stahlecker. Fuzzy prior information and minimax estimation in the linear regression model. Statistical Papers, 38.4:377-391, 1997.

[2] J. M. Bernardo. Bayesian statistics. Probability and Statistics (R. Viertl, ed) of the Encyclopedia of Life Support Systems (EOLSS), UK: UNESCO, 2003.

[3] A. Charnes, E. L. Frome, and P. L. Yu. The equivalence of generalized least squares and maximum likelihood estimates in the exponential family. Journal of the American Statistical Association 71, 353, 1976.

[4] A. Clauset. A brief primer on probability distributions. Santa Fe Institute http: //tuvalu.santafe.edu/ aaronc/courses/7000/csci7000-001_ 2011_L0.pdf, 2011. 
[5] Y. S. Erdogan and P. G. Bakir. Inverse propagation of uncertainties in finite element model updating through use of fuzzy arithmetic. Engineering Applications of Artificial Intelligence, 26.1:357-367, 2013.

[6] J. R. Fonseca, M. I. Friswell, J. E. Mottershead, and A. W. Lees. Uncertainty identification by the maximum likelihood method. Journal of Sound and Vibration, 288:587-599, 2005.

[7] M. I. Friswell and J. E. Motterhead. Finite Element Model Updating in Structural Dynamics, volume 38 of Solid Mechanics and its Applications. Kluwer Academic Publisher, 1995.

[8] W. R. Gilks. Markov chain monte carlo. John Wiley \& Sons, Ltd, 2005.

[9] T. Haag, S. C. González, and M. Hanss. Model validation and selection based on inverse fuzzy arithmetic. Mechanical Systems and Signal Processing, 32:116 - 134, 2012.

[10] T. Haag, J. Herrmann, and M. Hanss. Identification procedure for epistemic uncertainties using inverse fuzzy arithmetic. Mechanical Systems and Signal Processing, 24:20212034, 2010.

[11] P. C. Hansen and D. P. OLeary. The use of the l-curve in the regularization of discrete ill-posed problems. Journal of Computational and Applied Mathematics, 279:123-132, 2015.

[12] M. Hanss. Applied Fuzzy Arithmetic - An Introduction with Engineering Applications. Springer, Berlin, 2005.

[13] A. Howard and C. Rorres. Elementary linear algebra with applications. John Wiley, 2005.

[14] V. G. Ivancevic and T. T. Ivancevic. Geometrical dynamics of complex systems: a unified modelling approach to physics, control, biomechanics, neurodynamics and psycho-socioeconomical dynamics. Springer Science \& Business Media, 31, 2006.

[15] L. Jaulin and E. Walter. Set inversion via interval analysis for nonlinear bounded-error estimation. Automatica, 29.4:1053-1064, 1993.

[16] M. C. Kennedy and A. O'Hagan. Bayesian calibration of computer models. Journal of the Royal Statistical Society, 63:425-464, 2001.

[17] H. H. Khodaparast. A spectral approach for fuzzy uncertainty propagation in finite element analysis. Fuzzy Sets and Systems, DOI: 10.1016/j.fss.2013.10.005, 2014.

[18] H. H. Khodaparast, J. E. Mottershead, and K. J. Badcock. Interval model updating with irreducible uncertainty using the kriging predictor. Mechanical Systems and Signal Processing, 25.4:1204-1226, 2011.

[19] R. Kohavi. A study of cross-validation and bootstrap for accuracy estimation and model selection. IJCAI'95 Proceedings of the 14th international joint conference on Artificial intelligence, 2:1137-1143, 1995.

[20] E. L. Lehmann and G. Casella. Theory of Point Estimation (2nd ed.). Springer, 1998. 
[21] Y.-C. Lin and Y.-M. Huang. A fuzzy-based prior knowledge diagnostic model with multiple attribute evaluation. Educational Technology \& Society, 16.2:119-136., 2013.

[22] Y. Liu and Z. Duan. Fuzzy finite element model updating of bridges by considering the uncertainty of the measured modal parameters. Science China Technological Sciences, 55:3109-3117, 2012.

[23] T. Marwala. Finite-element-model Updating Using Computational Inteligence Tchniques. Springer Verlag, 2010.

[24] W. Oberkampf. Model validation under both aleatory and epistemic uncertainty. In Proc. of NATO AVT-147 Symposium on Computational Uncertainty in Military Vehicle Design, Athens, Greece, 2007.

[25] S. Y. Park and A. K. Bera. Maximum entropy autoregressive conditional heteroskedasticity model. Journal of Econometrics (Elsevier), pages 219-230, 2009.

[26] I. V. Tetko, D. J. Livingstone, and A. I. Luik. Neural network studies. 1. comparison of overfitting and overtraining. Journal of Chemical Information and Modeling, 35.5:826833, 1995.

[27] E. W. Weisstein. Median. From MathWorld-A Wolfram Web Resource. http://mathworld.wolfram.com/Median.html.

[28] G. Zyskind and F. Martin. On best linear estimation and general gauss-markov theorem in linear models with arbitrary nonnegative covariance structure. SIAM Journal on Applied Mathematics, 17.6:1190-1202, 1969. 